\title{
Are Emojis Creating a New or Old Visual Language for New Generations? A Socio-semiotic Study
}

\author{
Hamza Alshenqeeti \\ School of Arts and Humanities, Taibah University, PO Box: 344, Madinah, Saudi Arabia \\ E-mail: hshenqeeti@taibahu.edu.sa
}

Doi:10.7575/aiac.alls.v.7n.6p.56

URL: http://dx.doi.org/10.7575/aiac.alls.v.7n.6p.56
Received: 16/08/2016

Accepted: 05/10/2016

\begin{abstract}
The increasing use of emojis, digital images that can represent a word or feeling in a text or email, and the fact that they can be strung together to create a sentence with real and full meaning raises the question of whether they are creating a new language amongst technologically savvy youth, or devaluing existing language. There is however a further depth to emoji usage as language, suggesting that they are in fact returning language to an earlier stage of human communication. Parallels between emojis and hieroglyphs and cuneiform can be seen which indicates the universality of visual communication forms, rather than written alphabetised language. There are also indications that emojis may be cultural or gender-specific with indications that women use more emojis than men to express their feelings and that age is less of an indicator of usage than technological awareness and capability. It appears that emojis are filling the need for adding non-verbal cues in in digital communication about the intent and emotion behind a message. Examinations of the way that emojis have developed and evolved and their current and forecast usage leads to the conclusion that they are not a "new" language developed by the technological adept younger generations, but instead are an evolution of older visual language systems that make use of digital technology to create greater layers and nuance in asynchronous communications. Furthermore, emojis are devices for demonstrating tone, intent and feelings that would normally be conveyed by non-verbal cues in personal communications but which cannot be achieved in digital messages. It is also evident from prior works and analyses of usage that there are universal meanings to Emojis. This suggests that as a language form, emojis may be able to contribute to increased cross-cultural communication clarity. Further research is however recognised as being necessary to fully understand the role that emojis can play as a visual language for all generations, not just those termed millennials or technologically savvy youths.
\end{abstract} Keywords: Emojis, socio-semiotic analysis, new language, old language, pictograms

\section{Introduction}

Communication between humans is constantly changing and adapting to social trends, lifestyles and more recently technology, and language is recognised as being a living organism (Jesperson, 2013:7). Language responds to social change and attitudes (Meyerhoff, 2011), and its forms and usage evolve according to the needs of its users and the tools they can access for communication (Crystal, 2001:70). The rise of mobile communication devices initially raised concerns from traditionalists in the linguistic community and elsewhere that language was becoming terse and short and vital communication cues, particularly non-verbal ones were being lost, devaluing overall communicative ability (Tayebenik and Puteh, 2012). There is, however, a counter-argument which recognises that language comes in many forms, and one of these is the use of emojis and emoticons. In particular, how they provide greater nuance and clarity to text messages and are an expression of creativity in language, indeed as McGrath (2006) notes for some users there is a level of exhibitionism and demonstration of prowess in how they can construct phrases and meaning just with emojis. One group in particular, those who are classed as Millennials (i.e. born after 2000) are heavy users of these devices and this raises the question of whether emojis are creating a new visual language for a new technologically savvy generation or whether they are in fact simply revitalising a much earlier form of expression in the digital realm. Verbal languages were an evolution of early pictorial or symbol languages and there are clear parallels that can be drawn between these and emojis. The focus of this paper is therefore to consider the growth in the use of emojis and what this means for language and communication development in the $21^{\text {st }}$ century. This is an important area of language and communication development, illustrated by the fact that the Oxford English Dictionary selected the emoji for "tears of joy" (-) as its "Word of the Year" for 2015, showing how frequently it is used in communication (c.f. Fullwood et al, 2015). An initial focus is to examine the evolution of these symbols and the alignment of these with early communication forms that were not reliant on today's alphabet based systems. 


\section{Evolution of Emojis and Emoticons}

Emojis are often confused and used interchangeably with another digital symbolic form, emoticons. However, there are some clear distinctions. These are keyboard symbols which are combined to make pictures, for example ":)" which represents a smiley face, and " $<3$ " which represents a heart (Novak et al, 2015). The distinction between these keyboard stroke created symbols and emojis is that the latter are small pictures, first introduced by Shigetaka Kurita in Japan in the late 1990s to provide contextual cues and emotional context on a mobile internet platform (Skiba, 2016). Indeed, the term 'emoji' comes from the Japanese character (絵, "picture") + moji (文字, 'character'), and not as is commonly believed from the English word emotion (Skiba, 2016). These tiny images, initially designed for one platform were quickly adopted by Japanese mobile device users as a means of expressing emotions in text and other digital information, before spreading to the whole world, and as Moschini (2016) notes, are seen as an increasingly rich form of communication and as a manifestation of the playfulness and visual nature of digital culture.

Since the initial face-based emojis were introduced, the potential the library of emojis has grown and evolved and now includes animals, food, faces and other pictures as per figure 1 which can be used individually or together to create meaningful communicative strings.

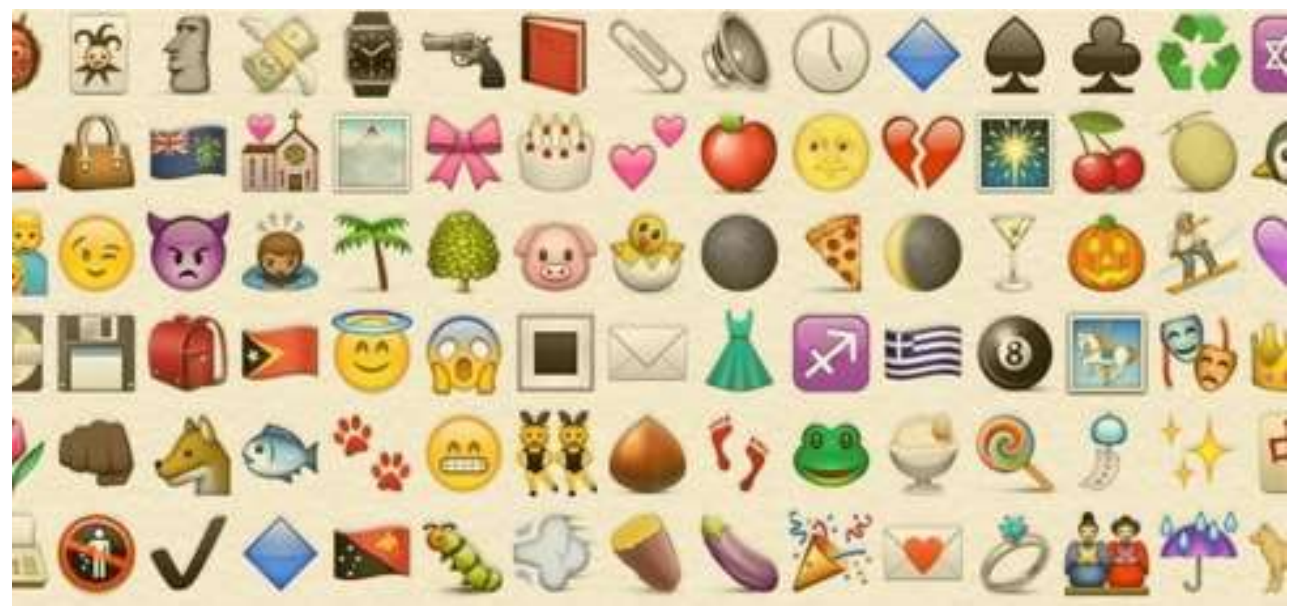

Figure 1. Common emojis (Ozaiza, 2015)

The illustration above shows how different digital pictograms (the emojis) are able to convey information without the use of morphemes and other grammatical structures (Lucas, 2015). Emojis can be inserted individually or together to create a string. These visual representations of words or constructs deliver enhanced meaning to the messages being sent in a creative and highly expressive format (Tauch and Kanjo, 2016). In certain quarters, there is a belief that the increased use of emojis is a sign that language is devolving, and returning society to the pictorial representations of ancient history which are deemed less intellectual or intelligent (Jones, 2015; McIntyre, 2016). What the detractors of emojis do not acknowledge is that emojis are a sign of the evolution and living nature of language (Goldfield, 2012). In addition, the complexity of these early pictorial systems is also contradictory to the devolution of language perspective (Adler et al, 1991). What is particularly interesting when looking at emojis is the correlation between 40,000 year-old cave paintings which told stories in pictures using humans, animals and other images rather than words, and in the Egyptian hieroglyph system of communication, used to record history (Scoville, 2015), as seen in the figure below.

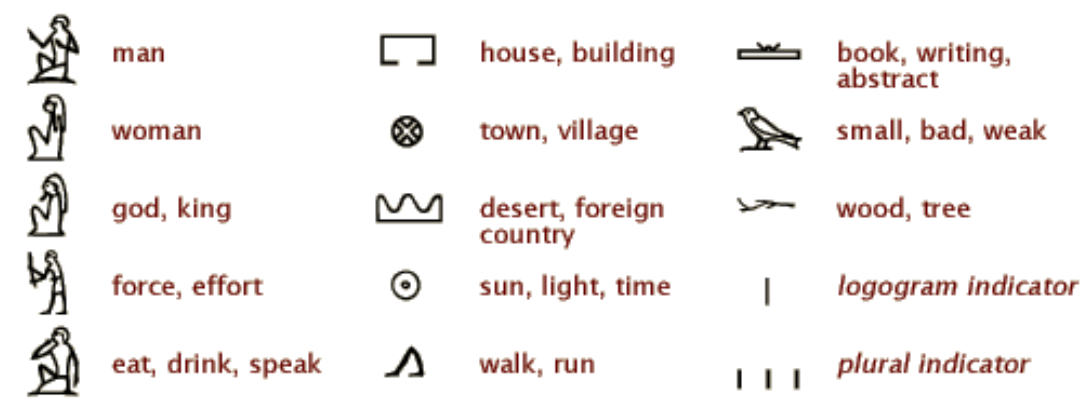

Figure 2. Egyptian Hieroglyphs

Emojis for "man", "sun" show clear parallels to this pictographic language as the following figure indicates: 


\section{$\lambda$ 婃}

Humans have always needed to communicate, and in particular, communicate their feelings and emotions to one another, either as a protection or sharing device and throughout history have used pictures or symbol to achieve this and create visual connections with one another (Fischer, 2003). Critics of emojis also cite that when texting first became popular, the use of shortened text language was popular, creating concern about a loss of literacy and communication skills (Crystal, 2001). However, there does not exist concrete and agreed findings that this has been the case, as Winzker et al, (2009) note, and indeed the use of Emojis is now more frequently seen as a highly creative form of language (Moschini, 2016). Messages created with emojis can be highly advanced and complex, and also reflect as Grace (2015) notes, a return to the notion of "rebus", which is from the Latin "not with words but with things". Indeed, not only the Egyptians but da Vinci and even Lewis Carroll, the author of Alice in Wonderland, used pictograms or visuals when writing to as they considered it not only creatively expressive, but also effective as a means of conveying ideas (Abujaber et al, 2012). The illustration in figure 3, a letter from Lewis Carroll illustrates this very clearly, and demonstrates how today's Emojis reflect earlier pictorial insertions in written language to convey richer meaning and connection.

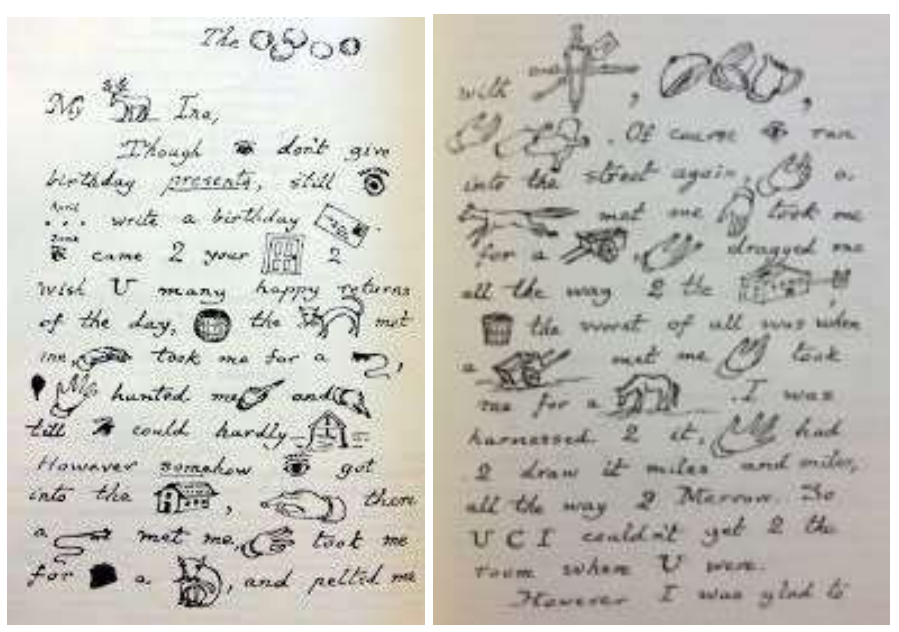

Figure 3. Rebus Letter from Lewis Carroll (The American Reader, 2016)

As the figure illustrates, pictures provide a greater level of depth and personalisation to the letter as well as the feelings of the writer. What is particularly striking is his use of " $\mathrm{U} \mathrm{C} \mathrm{I"} \mathrm{which} \mathrm{is} \mathrm{reflected} \mathrm{in} \mathrm{current} \mathrm{text} \mathrm{language,} \mathrm{as} \mathrm{is} \mathrm{his} \mathrm{use}$ of the "eye" drawing to signify "I" and a smiley face at the end and a "deer" image to indicate the initial salutation at the start of the letter. These symbols and representations are all clearly evident in the Emoji use seen in today's text messages. (Brisson, 2015). What this highlights is that Emojis, far from being a devaluation of language, are in fact an evolution of earlier pictographic and hieroglyphic language and not an entirely new invention.

Indeed, it can be argued that emojis are in fact expanding linguistic ability, and opening up new possibilities for innovative communication channels and expansion of traditional writing, making language more visual and playful and thus returning to more creative forms of language (Danesi, 2016). There are clear echoes of the past here, if one looks at the precursors of modern writing systems, such as the hieroglyphs shown above and other pictographic systems of communication. However, one of the challenges of accepting Emojis as a language form in their own right and as a complement to alphabetic systems is thatthat the widespread use of Emojis evolved from teen and commercial culture, and initially there was a wide and divergent range of images used, due to the different networks and platforms (Thurlow and Brown, 2003). However, both Google and Apple recognised the value of standardising the symbols and in 2010 using the Unicode Consortium, over 722 emoji codes were standardised (Lucas, 2016). This has parallels to the attempts at standardisation of typefaces in the 16th century, so that engravers no longer had complete freedom over how their letters were produced, and which led to a revolt against control and management of what was an evolving language (Baddeley and Voeste, 2012,p.175). The same can be said for Emojis, which whilst generally standardised are not all Unicode approved and may have meaning to only one small group or population, much like regional variants of spoken and written language. Again, this underlines the role of Emojis as language as frequently social groups develop their own paralanguage to identify themselves and their peers as being different to the mainstream. It is these paralanguages that lead to the evolution of language over time, and certainly this has been the case for Emojis, further strengthening the argument that they are an old language adapted to a new society and to meet challenges of message size, bandwidth speed and texting constraints (Gamble and Gamble, 2016). Emojis can therefore be considered as an expedient way to convey pragmatic information in a short text through imagery, much as ancient pictographic communication systems did (Kern, 2015). In addition, emoji language is not something which can be taught, rather it is acquired over time, through use and sharing. Emojis have emerged as a means of indicating euphemisms, sarcasm, 
hints and affection which were previously difficult to convey within a text and their visual nature is part of longer tradition of using images to convey meaning in writing (McIntyre, 2016). The rebus letter of Lewis Carroll shown above is one example of this.

At the same time, communication is complex and the use of emojis can either clarify or confuse the receiver, because they indicate presence of emotion but absence of the individual, due to their digital nature. In addition, there remains a level of interpretation, the smiley face emoji can mean "I'm happy" or "I like this", depending on the focus of the message, the relationship between the interlocutors and the context of the message (Dresner and Herring, 2010). This is a further parallel with alphabetised language and reinforces the multiples roles that emojis may take in enhancing communication. At the same time, it also underlines that emojis are not a new language, solely for the use of a younger generation, but that they represent a return to the use of visualisation, and thus coding of information. As Hagy, (2010, 354) notes, the vast array of information that the world offers today can now be condensed and made concise and clear, through the use of visualisations such as emoticons and emojis. As with cave paintings, cuneiform and other pictographic language systems or other visual deliveries of information, such as art, the interpretation and understanding provide clues to meaning and intent of the author, as well as feelings and emotions (Hagy, 2010, 355).

A further valuable facet of emojis is that they may be universal in nature, and thus can potentially be used for individuals to communicate even if they do not share a common verbal language as they provide hints as to intent and tone (Gulsen, 2016). For instance, if the letter written by Lewis Carroll is considered in terms of tone, it is apparent that the writer is cheerful and has warm feelings to the recipient. This suggests again that emojis, rather than being a new language, are in fact an old language that has been adapted to advances in communication technology and can deliver a more universal mode of communication than any alphabetic based language system. This has been recognised with new networks and increasingly creative use of Emojis.

A new mobile texting network called emojli was launched in 2014, which allows users touse emojis exclusively for communication. Whilst dismissed by some as a fad, its existence suggests that it is recognising the natural form of communication for humans (Hern, 2014). Certainly ass a species, humans have communicated with images far longer than we have with words, using icons, pictograms and other visual formats, as Lester, (2013,p.70) notes, with posters, symbols and other visual means providing the majority of mass communication until the invention of the printing press, all of which provide a clear genealogy for the wide appeal and rapid adoption of emojis. Comparing emojis to some of the earliest visual communication systems underlines this view.

The earliest pictograms were those of cavemen, who used images to create abstract ideas about their lives, their stories and their beliefs and feelings. Even the earliest writing systems such as Cuneiform, used by the Sumerians in 3300 (BC) was a system of icons made by tools on wet clay, which evolved as the technology and tools of the period advanced. This is again a parallel with the development of emojis which are only possible due to advances in digital programming technology as Tuttle, (2016 p.62) notes. The figure below shows the evolution of cuneiform from literal to abstract symbols, similar to that seen in the development of early to the later more sophisticated emojis in use today.

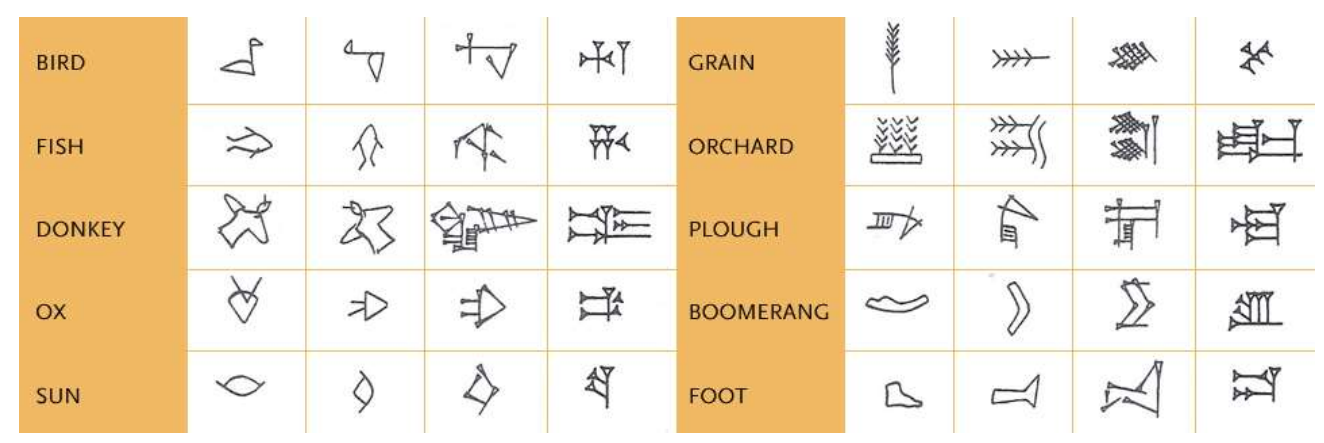

Figure 4. Cuneiform Evolution

Looking at these symbols and the common emojis, digital emojis can be seen as descendants of these universally recognised icons or pictograms. Similarly, these symbols were originally used as stand-alone depictions but as the need to keep records increased, strings of symbols came to have a particular meaning. The same has happened with emojis which can now be placed in sequence to create a particular meaning as the figure below, and the additional items in the appendices indicate:

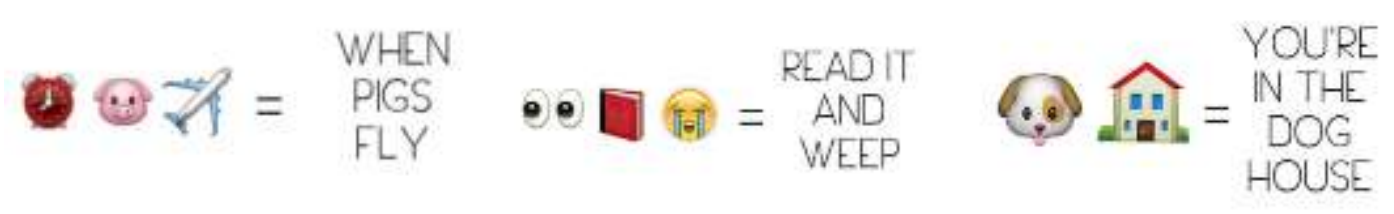

Figure 5. Emoji Strings (Alldred, 2014) 
Cuneiform and other symbolic forms of written communication however were gradually replaced by standardised letters which eventually, with the aid of the printing press in the 15th century, became the standard grammar, punctuation and written language used today (Lester, 2013, p.72). However, visual or pictorial communication has remained in the background, as the rhebus letters from Lewis Carroll shown in figure 3 underline (Hagy, 2010, p.62). When considering the evolution of emojis however, it should also be noted that emojis originated in Japan, who, along with the Chinese share a pictorial language format and thus the parallels with their character systems are potentially closer. Certainly, looking at its' antecdents, the emoji is more universal and focused on emotions and conveying a deeper intent than can be achieved through the written (alphabetised) form (Azuma, 2012). Given this it is pertinent to consider the role of emojis as non-verbal communication devices.

\section{Emojis as non-verbal communication}

Spoken language, in any tongue is nearly always delivered with emotional or physical cues, whether this is voice tone, hand or eye gestures and other visual elements. In a digital context, these are not present. Emojis close this gap in a creative and highly visual way and ensure the writers thoughts and feelings are expressed in a concise and fun way. Given that many facial expressions of emotion are universal this also underlines the potential universality of the emoji language (Azuma, 2012). In effect, they act as non-verbal surrogates, telling the reader of the message what the facial expression of the writer would be, and delivering additional social cues to support understanding of the message.

Natural language is rarely accompanied by speech without emotional context or physical gestures, making it multimodal. Emojis help writers convey thoughts and feelings digitally by filling the void for facial expressions that enhance verbal communication. Charles Darwin, the famous anthropologist, found that facial expressions of emotion are universal, not learned differently in each culture. He concluded that all humans are anatomically alike and use their muscles similarly to express feelings (Mesquita et al, 1997, p.257). Subsequently, common facial expressions are often universal, and the emojis that represent them can be used to communicate emotion universally. This global understanding of pictographic representations of emotions thus can clearly contribute to effective cross-cultural communication. This is a further parallel to the Egyptian hieroglyph system which was understood across all the regions controlled by the Pharaohs, irrespective of their social status (Coulmas, 2003). In this respect, the universality of emojis was recently assessed by Instagram and the figure below indicates the usage and frequency of these icons across a diverse range of countries.

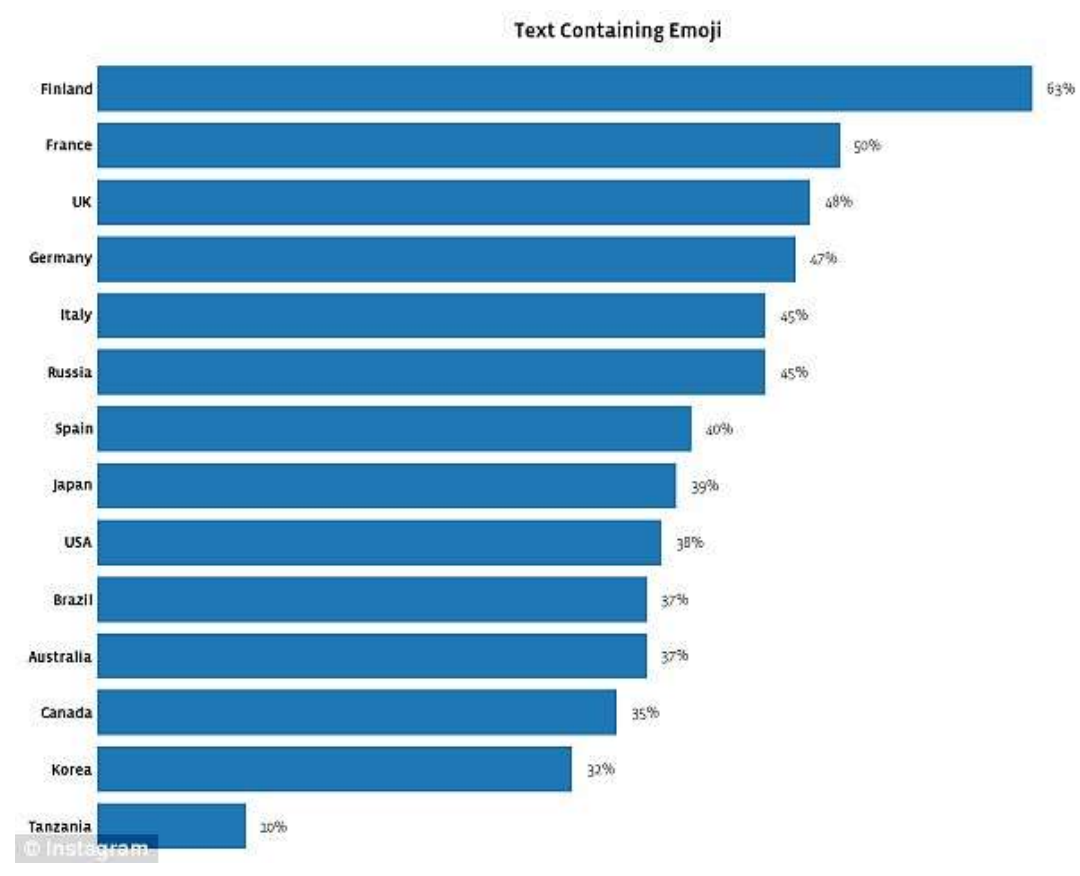

Figure 6. Emoji use by country (Woollaston, 2015)

In many Western countries, as the figure above indicates, nearly $40 \%$ of all texts contained at least one emoji, and even in countries such as Korea, one third contained these symbols. Additional data on frequency and use of emojis can be found in the Appendices. Recognising this growth and the potential of emojis as a language form, Azuma and Maurer (2007) considered whether this increased usage of emojis meant a new or an auxiliary language for digital communication. Their findings indicated that emojis would develop into a universal symbolic language and certainly the increasing range of emojis and their frequency of use appears to corroborate this view. One of the reasons for the popularity of these small symbols is their ability to express emotional complexity in a short and creative way, that cannot be conveyed with verbal language, especially in the written form as inflection, tone and rhythm. This suggests, as Crystal (2008) notes that emojis, and indeed text language are not devolving language but rather enhancing both literacy and emotional expression as well as demonstrating the evolution of language as a living entity that responds to social and cultural change. For millennials therefore, emojis and indeed text language can be 
seen as new form of writing, the rise in blog posts, tweets, and instantly available information on other social medium is an illustration of how widespread this language form has become. These new genres of writing are challenging the status quo of traditional literary compositions and this may be one of the causes of concern for traditionalists (Crystal, 2008). However, as already noted language is not stagnant, it evolves with society and new forms and terminologies are constantly appearing, and emojis are just another aspect of this. Pulling away from the restrictions that alphabet systems place on creative expression, millennials use emojis as social-emotional communication, offering new layers of understanding and expression in a visual rather than orthographic format. Returning again to the parallels and connections between hieroglyphs or Chinese character writing, these can mean different things depending on the characters or pictograms in the string. This is the same for emojis and illustrates how they underline tone and meaning as a new version of an old language and communication format (Brisson, 2015). What this leads to is to consider how emojis can either replace or enhance language, as a way of reviewing the initial question of whether they are a new or an old language form.

\section{Emojis as a Language Form}

As already noted, written text is missing non-verbal cues and emojis can provide these. However, there is an argument, as Dresner and Herring (2010) suggest that the role of these visuals does not incorporate actual linguistic traits of English as they do not contain letters. In effect, if emojis do not have a direct convention and connection to English for example, can they really be viewed as a language?. The answer to this lies in the fact that the user of an emoji must have a certain understanding of the rules of English, in terms of tone and socio-cultural/pragmatic intent in order to determine which emojis best meet their desired context and intent. In this respect, the conventions of speech theory still apply, suggesting there is a language base for emojis (Kavanagh, 2016). In addition, emojis can be seen as similar to logographs, as they may be representative of a singular morpheme, or word, but one image may be used to indicate multiple emotional reactions. As such they are a language, but one with universal concepts (Brisson, 2015) and thus have a wider comprehension potential amongst different cultures.

At the same time, speech act theory indicates that speech acts are not just communicative acts but also indicative of the state of situation, the emotions of the speaker and listener and emojis can take a role non-verbal indicators of the emotional context of a message, acting as a bridge between verbal and written speech acts (Dresner and Herring, 2014). What this suggests is that in future emojis are likely to be more commonplace than verbal texts and even emails, highlighted by the Oxford English Dictionary's "word of the year" noted in the introduction. In this regard, emojis are clearly a language form, but one which is related to older, more naturalistic forms of communication than the written word, which in fact separated those who could read and write, from those who could not. Emojis, as a language have reclosed this cap, underlining again that they are an old, resurfaced form of language Lucas (2016).

Indeed, emojis offer new possibilities in terms of communication. Their application as a code system that can complement and augment the tone and visual vocabulary of text messages is likely to be continuous and unrestrained. Unlike a standard alphabetical system, which generally starts with a signifier (for example describing an object such as "house") to imply a signified item (a specific house), emojis offer the potential to share a set image which implies connection to the house, how they feel about it and what it means in the message, with one or two pictorial representations, rather than long drawn messages of explanation. In this regard as Lee $(2016,208)$ notes, they are enhancing the written word rather than completely replacing it. In addition, emojis can be grouped to increase emphasis, and create responses of greater creativity and imagination than words alone. As such, they are demonstrating their connection to earlier language forms, but in a more sophisticated, technologically coded manner (Brisson, 2015).

There are however some generalisations about the use of emojis as language, with suggestions that women are using them more than men (Dresner and Herring, 2010); and men being more likely to use sarcastic emojis and perceptions of their usage as positive or negative being generation dependent. However, when considering the future of language, and whether emojis are a new language form, it is also clear that millennials are less rule-bound than previous generations, and are more likely to include emojis in their texts and even work emails. Does this reinforce the notion of emojis as a new language form or does it simply recognize the evolutionary nature of language and the desire for visual information that is part of the human condition? (Hagy, 2010).

\section{Generational and Cultural Variations in Emoji usage}

In the same way that language is a constantly evolving phenomena and is subject to social, generational and cultural variations, so too is technology and how it is used. Those who have grown up with text language, mobile technologies and digital mediums are frequently more accepting of changes to the way these operate, for example incorporating emojis into their messages (Krohn, 2004). However, as Nishimura (2015) notes, these generational differences are not as stark as one might expect. Rather they appear to grounded in the exposure to and experience of technology of the individual within their own environment. Those in older generations who are exposed to technology, either through their work or their children/grandchildren are more accepting of changes such as increased use of emojis. Indeed, as Oakley (2016) notes, even the 90 year-old Monarch of the UK, Queen Elizabeth uses social media such as Twitter and may even use emojis with her younger family. This highlights that language in visual form such as pictograms, logograms and symbols, all terms that have been employed to describe emojis, can traverse generational as well as cross-cultural boundaries. 
Furthermore, in the same way that verbal interactions will cover a range of subjects and emotions, dependent on the social context and conventions of the interlocutors, emojis can also be sub-divided into different groups as Nishimura (2015) notes. His work on the various demographics of emoticon use is one of the most comprehensive to date, and takes into account the following non-linguistic symbols that may appear in texts or email and are based on descriptions by Azuma and Ebner, 2008).

- Typographic face marks, for example (:)) "smile" and (:() "sad face" which are lines used to create facial expressions.

- non-linguistic symbols, such as 5$\} \odot$. They often appear at the end of a sentence and may function as punctuation, but with more visual impact and added meaning.

- Emoji or colourful graphics representing faces, objects and ideas. These may be animated such as beating hearts or blinking stars. They can function lexically to replace words as in the rebus letters indicated above, for example the "II " to indicate the word beer.

Examining data from 200 blogs Nishimura identified that not only did women tend to use a higher number of emojis, as the figure below indicates, but that there was not a major variation between the age groups and in fact older females seemed to use more than younger.

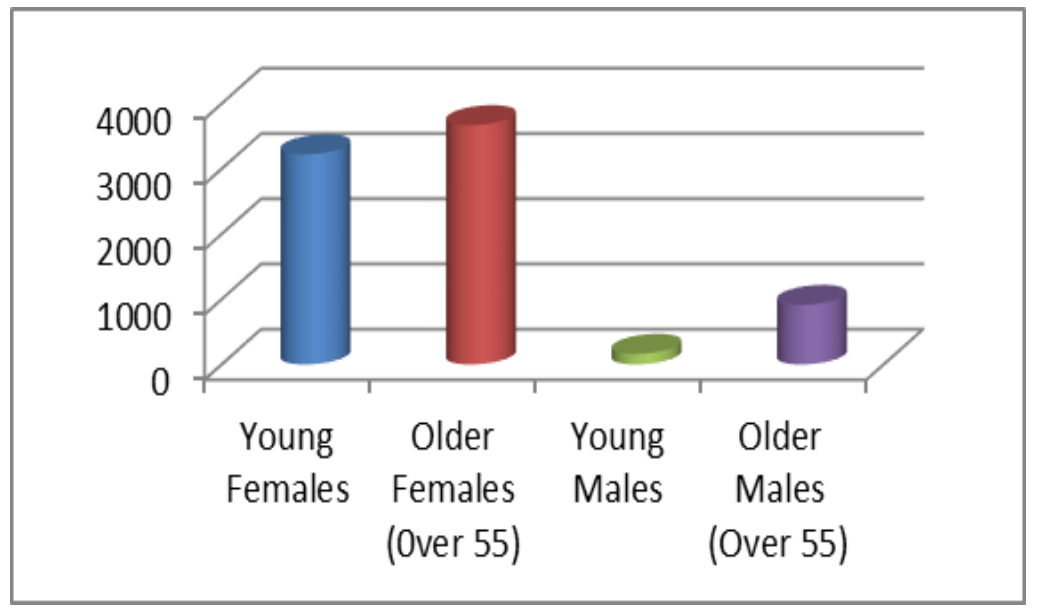

Figure 7. Use of Emojis/Emoticons Across Generations and Genders (Nishimura, 2015:5)

A similar picture emerged when looking at frequency of emoji use over the 200 blogs as the figure below indicates. Again, the older females had the highest usage and women used nearly three times the number of emojis as the men in the study.

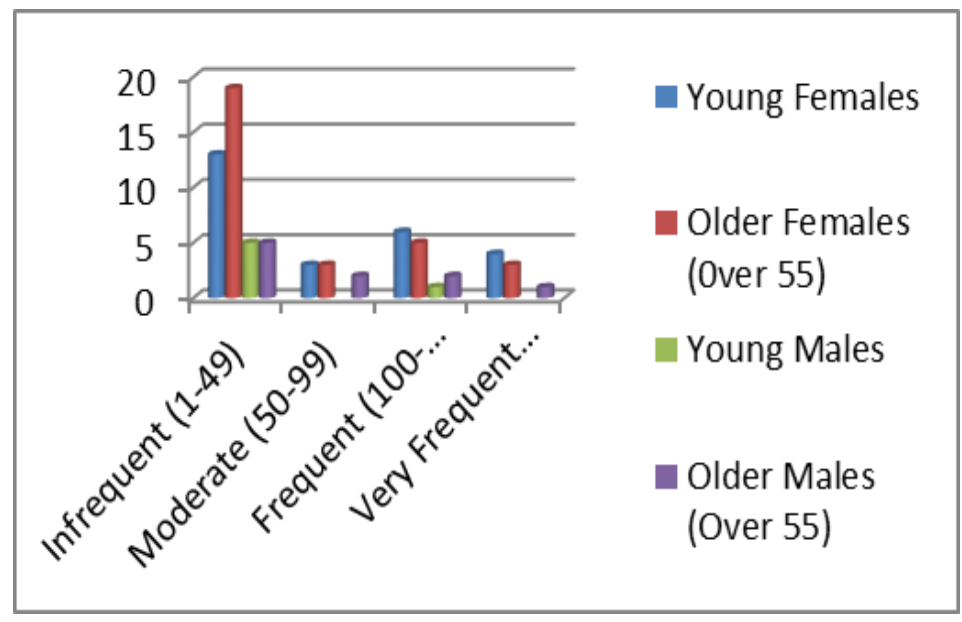

Figure 8. Frequency of Emoji use (Nishimura, 2015:5)

It should be noted however that Nishimura's study was conducted in Japan and therefore other, cultural factors may play a part in his findings. However, an analysis by Kika, an emjoi keyboard application for Android indicated that there are cultural variations in the use of emojis. As Lu et al, (2016) note, the fact that this keyboard has been developed specifically for users to utilise the images to send non-verbal messages further underlines the function of emojis. In terms of cultural variations, the figure below indicates the most commonly used emojis across a range of country's assessed by Kika. 


\begin{tabular}{|c|c|c|}
\hline & \% emoji-msg & Top emoji \\
\hline US & 9.2 & 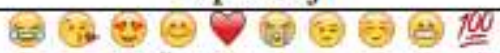 \\
\hline Brazil & 5.1 & G \\
\hline Mexico & 7.9 & 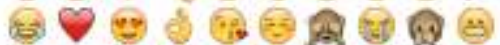 \\
\hline France & 19.8 & 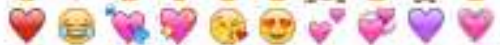 \\
\hline Spain & 3.4 & 붕 \\
\hline Turkey & 5.8 & 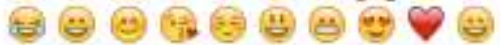 \\
\hline Argentina & 3.1 & 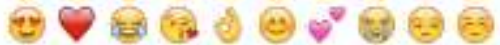 \\
\hline Indonesia & 3.2 & 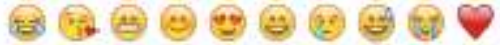 \\
\hline Russia & 10.9 & 엉 웅 \\
\hline Colombia & 3.7 & 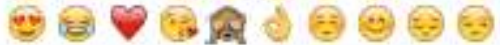 \\
\hline
\end{tabular}

Figure 9. Most Common Emojis by Country (Lu et al, 2015:774)

As the figure indicates, there are clearly some universal trends in the use of emojis, although frequency of usage varies a little from culture to culture. The Kika study also identified, in line with Nishimura (2015) that females were more likely to use emojis than males, and that younger users were also more prolific in their adoption of these symbols in their text messages, as the figure below indicates.

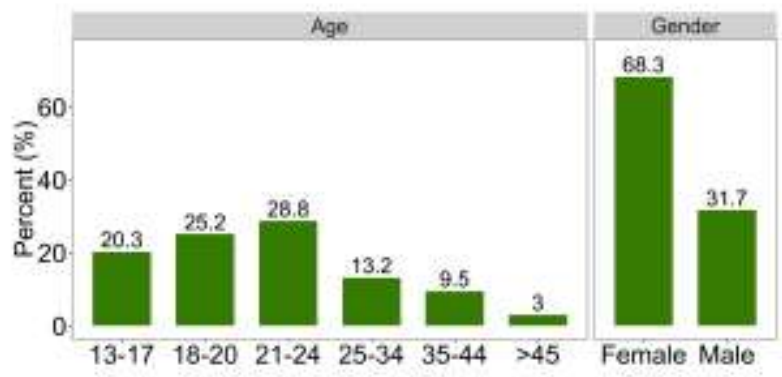

Figure 10. Gender and Age Distribution of Kika Emoji Keyboard Users (Lu et al, 2015:773)

Evidence from Nishimura (2015) and $\mathrm{Lu}$ (2016) confirms the over-riding indication that emoji use, as a form of language is both universal and cross-generational indicating that it is both recognised and accepted. What is also notable in Nishimura's study is that emojis were the most commonly used non-linguistic device when compared to the alternative emoticons examined. This further underlines their usage as distinctive language devices rather than simply additions or aesthetic accompaniment to the main content of the message. In addition, cultural variations in the popularity and frequency of emoji use have also been identified,

There is however another facet to the use of emojis that could detract from these initial assumptions of their usage as a distinct new or resurfacing language. This is that the focus is on emotions and feelings expressed pictorially. Given this, the role of emotions in language and how emojis reflect this within digital messages needs consideration as part of the understanding of whether emojis are a new or old language form.

\section{Emojis as Sentiment and Expressions of Emotion in Digital Messages}

The wide usage of emojis and their increasing popularity in tweets, blogs, and text messages across a range of cultures and ages suggest that users consider them as an effective and powerful tool for communicating feelings and emotions in a digital medium. This suggests users want to express sentiment and emotion in a clear and coherent way using new mediums. Novak et al, (2015) therefore undertook to develop an Emoji Sentiment Ranking that would identify how frequently particularly emojis are used, and thus sentiments expressed, in a sample of 1.6 million tweets from 13 European countries, it was found that $4 \%$ contained emojis. The connection of this approach is thatto understanding emojisis that sentiment analysis and opinion mining are important new areas in the field of language processing and as such are positively correlated to both the increased use of emojis but also their place as a new or evolved element of language and communication (Liu, 2012, 2015). In particular with whether the emoji is expressing a positive or negative sentiment. The work of Novak et al (2015) has identified that unlike the earlier emoticons, emojis may be placed anywhere within a text, not just at the end, but typically first appear around $2 / 3$ of the way through a text, although this varies depending on size of message. At the same time, the role of the emoji is not just to convey simply positive or negative overtones, but also more specific emotions and sentiments like as anger, happiness, or sadness, and there is evidence of them being used to define activities, locations, or objects of interest. All of these facets of emoji use underline the fact that their usage is clearly a language form. However, the argument stands that it is a form of visual language that is returning human communication to earlier modes that predate alphabetic and linguistic communication and language modes. 


\section{Summary and Conclusions}

This paper set out to examine the use and place of emojis as a form of language. In particular to determine whether these digital pictograms (also known as logograms or ideographic symbols) were a new language developed by a new technologically competent generation, or whether they reflected a deeper human need for visual communication that had been expressed through pre-alphabet communication systems. A brief review of earlier visual communication methods identified that pictographic representations of objects and sentiments have a correlation to and thus are ancestors of the emojis that are universally used today. Their representation of everyday objects and feelings through a visual format can be seen in numerous early language systems that began as visual depictions of the world around the users. Furthermore, the development of emojis from single images to deeper meaning through combinations or strings that remove the need for any text at all, can also be seen in the development of Sumerian Cuneiform and Egyptian hieroglyphics over times. This underlines that the "emoji language" is not a new language, but rather a new form of an old method of communication. In this respect, it can be argued that emojis are in fact a form of paralanguage, offering users a means to communicate with their own social groups in a form of code. It is this facet that has given rise to the view that it is for younger generations only but initial investigations into the area suggests that this is not the case,. rather it is technology acceptance and creativity that governs use of emojis, as well as, to some extent the complexity of the message.

Despite these strong positives for the use of emojis, there remains a perception in some quarters that their use is a lesser form of language and is devaluing and devolving language. However, these concerns are not only unfounded but they ignore human needs for non-verbal information within their communications. In addition, emojis are replacing internet slang such as "lol", as can be seen from the charts in the Appendices which was one of the main focuses of detractors of text language and supporters of the language devolution perspective. This ignores the fact that visual forms of language, which include non-verbal communication such as body language have been used since prehistoric times to underline and reinforce verbal language. Emojis therefore, are simply placing these visual forms into the digital arena. Text language, without emojis, can be misinterpreted in terms of context and meaning without additional clues. The emojis provide these clues in a creative and innovative way by delivering an indication to the receiver of the message of the sentiment to be expressed and the emotions behind the message. In addition, there is a further value to emojis which underlines that they are an evolution of old language forms. This is their universal nature. Although there are some cultural variations in the way that emojis are used, their increasing ubiquity around the world suggests that they may be a way to avoid, or at least reduce, cross-culture misunderstanding. The work has additionally identified that age is not necessarily an indicator of emoji use, although technology acceptance may be, and that women, who are naturally more expressive and emotional in their communication, are more prolific users than men of the emojis. There are however some cultural variations in the popularity and usage of certain emojis, despite a high level of universality. These would need to be examined in greater detail to identify what leads to these cultural variations in emoji use. Emojis can moreover, be considered to have a role as a language form in their own right and with a genealogy that dates back to the early language of humans depicted in cave paintings and other art forms that pre-date written information. Emojis, in essence are an expression of emotion and sentiment, and as a means of delivering creative non-verbal content in a digital medium are both old and new.

\section{References}

AbuJaber, H., Yagi, S.M. and Al-Ghalith, A., 2012. Spelling issues in EFL graffiti: Analysis and implications. European Scientific Journal, 8(21): 56-75.

Adler, R.B., Rodman, G.R. and Cropley, C., (1991). Understanding human communication. Fort Worth: Holt, Rinehart, and Winston.

Alldred, C., (2014), 21 Emoji Combinations To Use When Words Just Won't Cut it, [Online], available from http://blazepress.com/2014/10/21-emoji-combinations-use-words-wont-quite-cut/, [accessed 04.10.2016].

Azuma, J., 2012. Graphic Emoticons as a Future Universal Symbolic Language. Approaches to Translation Studies, 36: 61-84.

Azuma, J, and Maurer. H., (2007) "From Emotions to Universal Symbolic Signs: Can Written Language Survive in Cyberspace?" Institute of Information Processing and Computer-Supported New Media. Part of the Faculty of Science, Graz University of Technology.

Azuma, J. \& Ebner, M. (2008) A Stylistic Analysis of Graphic Emoticons: Can they be Candidates for a Universal Visual Language of the Future?. In J. Luca \& E. Weippl (Eds.), Proceedings of EdMedia: World Conference on Educational Media and Technology 972-979.

Baddeley, S., and Voeste, A., (2012), Orthographies in Early Modern Europe: A Comparative View, Amsterdam: Walter de Gruyter, 175: 1-14.

Brisson, C.M., 2015. Hieroglyphs at Our Fingertips. [Online], available from http://s3.amazonaws.com/academia.edu.documents/37726102/Hieroglyphs_at_Our_Fingertips_-

_Brisson.pdf?AWSAccessKeyId=AKIAJ56TQJRTWSMTNPEA\&Expires $=1475667176 \&$ Signature $=$ IvN8NDclwn9Ch ENOAxmEBf7\%2Fyho\%3D\&response-content-

disposition=inline\%3B\%20filename\%3DHieroglyphs_at_Our_Fingertips_Language_S.pdf, [accessed 05.10.2016]. 
Coulmas, F., (2003). Writing systems. An introduction to their linguistic analysis, Cambridge: Cambridge University Press.

Crystal, D., (2001). Language and the Internet, Cambridge, Cambridge University Press.

Crystal, D., (2008). Txting:The GR8 Db8, Oxford: Oxford University Press.

Danesi, M., (2016). The Semiotics of Emoji, London: Bloomsbury Publishing

Dresner, E. \& Herring, S. C. (2010). Functions of the nonverbal CMC: Emoticons and illocutionary force. Communication Theory, 20, 249-268.

Dresner, E. and Herring, S.C., (2014). Emoticons and illocutionary force. InPerspectives on Theory of Controversies and the Ethics of Communication(pp. 81-90). Amsterdam: Springer Netherlands.

Fullwood, C., Quinn, S., Chen-Wilson, J., Chadwick, D. and Reynolds, K., (2015). Put on a smiley face: Textspeak and personality perceptions. Cyberpsychology, Behavior, and Social Networking, 18(3): 147-151.

Gamble, T.K., and Gamble, M., (2016), Non-Verbal Messages Tell More: A Practical Guide to Non-Verbal Communication, London: Routledge.

Goldfield, H. (2012). "I Heart Emoji.” [Online],available from http://www.newyorker.com/culture/culture-desk/i-heartemoji, [accessed 04.10.2016].

Gülşen, T.T., (2016). You Tell Me in Emojis. In Ogata, T., Akimoto, T., (eds.). Computational and Cognitive Approaches to Narratology, New York: IGI Global, pp.356-378.

Hern, J., (2014), The Rise and Rise of Emoji Social Networks, [Online], available from https://www.theguardian.com/technology/2014/sep/12/emoji-social-networks-app-emojli-emojicate-steven, [accessed 09.10.2016].

Jespersen, O., (2013). Language: its nature and development. Oxon: Routledge.

Jones, J., (2015). Emoji Is Dragging Us Back to the Dark Ages - and All We Can Do Is Smile [Online], available from https://www.theguardian.com/artanddesign/jonathanjonesblog/2015/may/27/emoji-language-dragging-us-back-to-thedark-ages-yellow-smiley-face, [accessed 04.10.2016]

Kavanagh, B., (2016). Emoticons as a medium for channeling politeness within American and Japanese online blogging communities. Language \& Communication, 48: 53-65.

Kern, R., (2015). Language, Literacy and Technology, Cambridge: Cambridge University Press

Krohn, F.B., (2004). A generational approach to using emoticons as nonverbal communication. Journal of technical writing and communication, 34(4), pp.321-328.

Lee, J.S., (2016). Emoticons. In Ethical Ripples of Creativity and Innovation. Basinstoke: Palgrave Macmillan UK, pp. 207-213

Lester, P.M., (2013). Visual communication: Images with messages. Ohio: Cengage Learning.

Liu B. (2012). Sentiment Analysis and Opinion Mining. Synthesis Lectures on Human Language Technologies, 5(1):1167.

Liu B. (2015). Sentiment Analysis: Mining Opinions, Sentiments, and Emotions. Cambridge: Cambridge University Press.

Lu, X., Ai, W., Liu, X., Li, Q., Wang, N., Huang, G. and Mei, Q., (2016), Learning from the ubiquitous language: an empirical analysis of emoji usage of smartphone users. In Proceedings of the 2016 ACM International Joint Conference on Pervasive and Ubiquitous Computing (pp. 770-780).

Lucas, G., (2016), The Story of Emoji, London: Prestel Publishing

Marcus, A., (2003). Icons, symbols, and signs: Visible languages to facilitate communication, Interactions, 10(3): $37-$ 43.

McGrath, C., (2006). The Pleasures of the Text. New York Times Magazine, pp.15-19.

McIntyre, E.S., (2016). From Cave Paintings To Shakespeare And Back Again: What Are Emoji And Should I Be Afraid? (Doctoral dissertation).[Online], from https://digital.library.txstate.edu/bitstream/handle/10877/6100/McIntyreEmily.pdf?sequence=1, [accessed 09.10.2016].

Mesquita, B., Frijda, N.H. and Scherer, K.R., (1997). Culture and emotion. Handbook of Cross-cultural Psychology, 2: 255-297.

Moschini, I., (2016). The" Face with Tears of Joy" Emoji. A Socio-Semiotic and Multimodal Insight into a JapanAmerica Mash-Up. HERMES-Journal of Language and Communication in Business, (55), pp.11-25.

Nishimura, Y., (2015). A sociolinguistic analysis of emoticon usage in Japanese blogs: Variation by age, gender, and topic. In Selected Papers of Internet Research 16: The 16th Annual Meeting of the Association of Internet Researchers.

Novak, P.K., Smailović, J., Sluban, B. and Mozetič, I., 2015. Sentiment of emojis. PloS one, 10(12), p.e0144296. 
Oakley, N., (2016), The Queen Just Sent An Actual Tweet and Here's the Photo to Prove it, [Online], available from http://www.mirror.co.uk/tech/queen-just-sent-actual-tweet-8244651, [accessed 04.10.2016]

O'Brien, G., (2015), The Word on the Street Is not a Word, It's a ( 4 )", [Online], available from http://www.digitalamerica.org/the-word-on-the-street-is-not-a-word-its-an-grace-obrien/, [accessed 04.10.2016]

Ozaiza, O, (2015). How To Make Your iphone Tell You The Secrete Meaning of Emojis, [Online], available from: http://ios.wonderhowto.com/how-to/make-your-iphone-tell-you-secret-meaning-emojis-0148108/, [accessed, 07.10.2016.

Scoville, P. (2015), "Egyptian Hieroglyhs.” [Online], available from http://www.ancient.eu/Egyptian_Hieroglyphs/ [accessed, 04.10.2016].

Skiba, D.J., 2016. Face with Tears of Joy Is Word of the Year: Are Emoji a Sign of Things to Come in Health Care?. Nursing education perspectives,37(1): 56-57.

Tauch, C. and Kanjo, E., (2016), September. The roles of emojis in mobile phone notifications. In Proceedings of the 2016 ACM International Joint Conference on Pervasive and Ubiquitous Computing: Adjunct (pp. 1560-1565).

Tayebinik, M. and Puteh, M., (2012). Txt msg n English language literacy, Procedia-Social and Behavioral Sciences, 66: 97-105.

This Day in Lettres, (2016), [Online], available from http://theamericanreader.com/10-october-1869-lewis-carroll-togeorgina-watson/, [accessed, 14.10.2016]

Thurlow, C. and Brown, A., 2003. Generation Txt? The sociolinguistics of young people's text-messaging. Discourse analysis online, 1(1), p.30.

Tuttle, E.C., 2016. The Past, Present and Future of the English Language: How Has the English Language Changed and What Effects Are Going to Come as a Result of Texting? (Doctoral dissertation).[Online], available from http://scholars.indstate.edu/xmlui/bitstream/handle/10484/12084/Tuttle_Carlene_2015_HT.pdf?sequence=1\&isAllowed $=\mathrm{y}$, [accessed 09.10.2016].

Winzker, K., Southwood, F. \& Huddlestone, K. (2009). Investigating the impact of SMS speak on the written work of English first language and English second language high school learners. Per Linguam : a Journal of Language Learning, 25(2): 1-16.

Woollaston, H, (2015), Are Emoji Killing Off The Alphabet? [Online], available from http://www.dailymail.co.uk/sciencetech/article-3070135/Are-emoji-killing-alphabet-Instagram-maps-use-iconsreplacing-internet-slang-words.html, [accessed 04.10.2016].

\section{Appendices}

\section{Appendix I - Emoji Usage Growth and Statistics}

The figure below indicates the rise in use of emojis between 2014 and 2015.

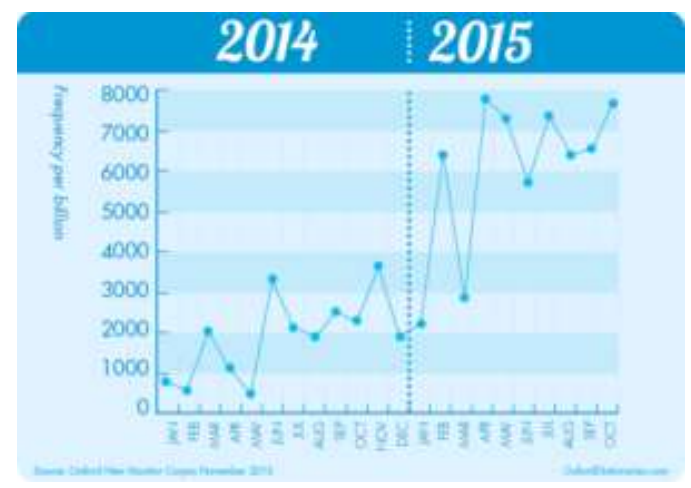

Figure 11. Emoji Usage 2014-2015 (O’Brien, 2015)

As the figure indicates, there has been a dramatic increase in the usage of emojis since early 2014. This is partly due to increasing technological capability which allows emojis to be used on Twitter as part of a hashtag, but also growth in smart phone usage and recognition of the value of emojis. The types of emojis used show some universal themes, but as the figure below indicates, there is still variation in which are the most popular for expressing sentiment. 


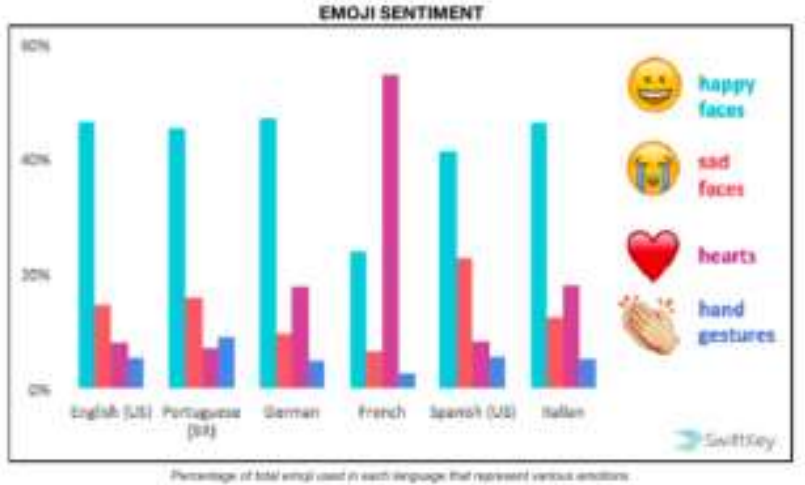

Figure 12. Emoji Sentiment Usage by Country

Source: http://digiday.com/brands/digiday-guide-things-emoji/ (2016)

In addition, emojis are replacing internet slang such as "lol" and "lmao" as the figure below highlights.

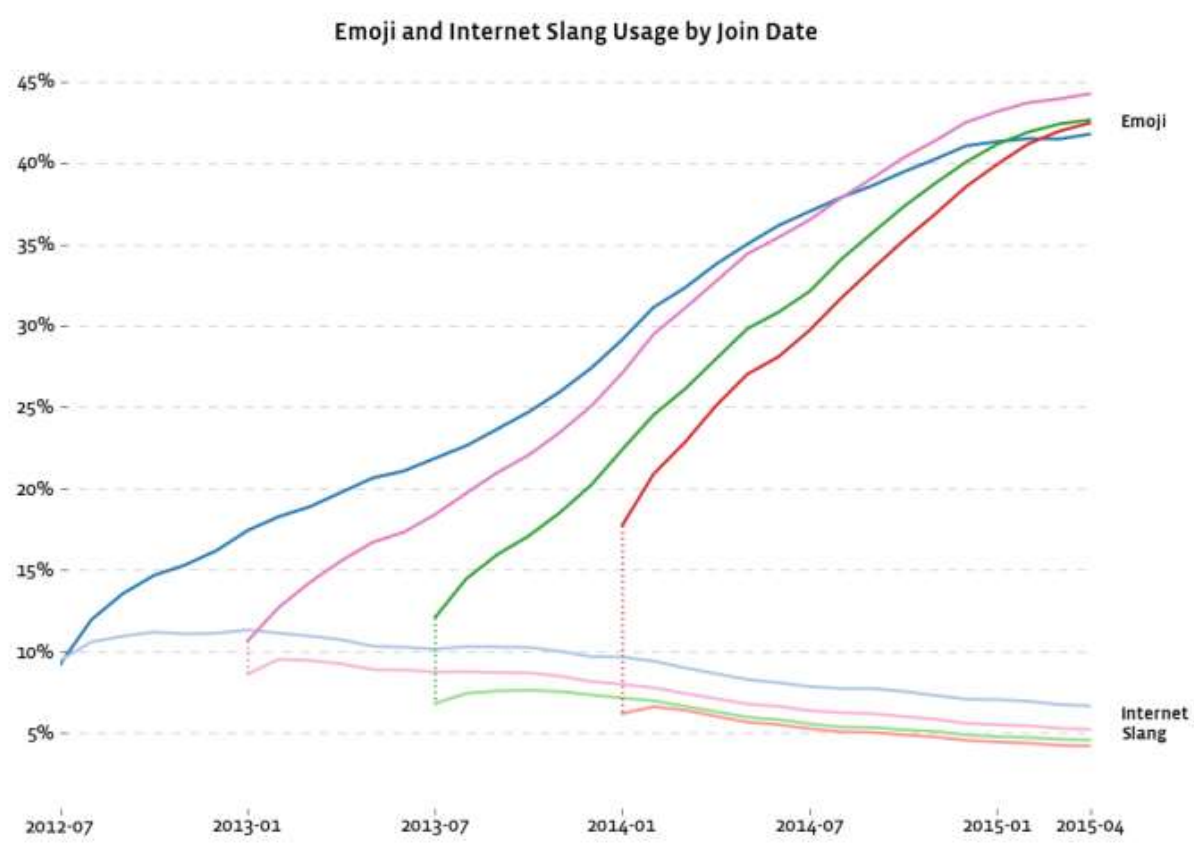

Figure 13. Emoji versus Internet Slang growth

Source: http://digiday.com/brands/digiday-guide-things-emoji/ (2016)

As the figure above indicates, there has been an exponential growth in the use of emojis with a corresponding decline in the use of internet slang, which appears to contradict the detractors of emojis who suggest they are devaluing language.

\section{Appendix II - Creative Use of Emojis}

As a paralanguage that conveys strong emotion, emojis can also be used to tell stories and string together information. The following examples demonstrate how this can be achieved. The first figure shows the plot of Les Miserables written out in emoji. 


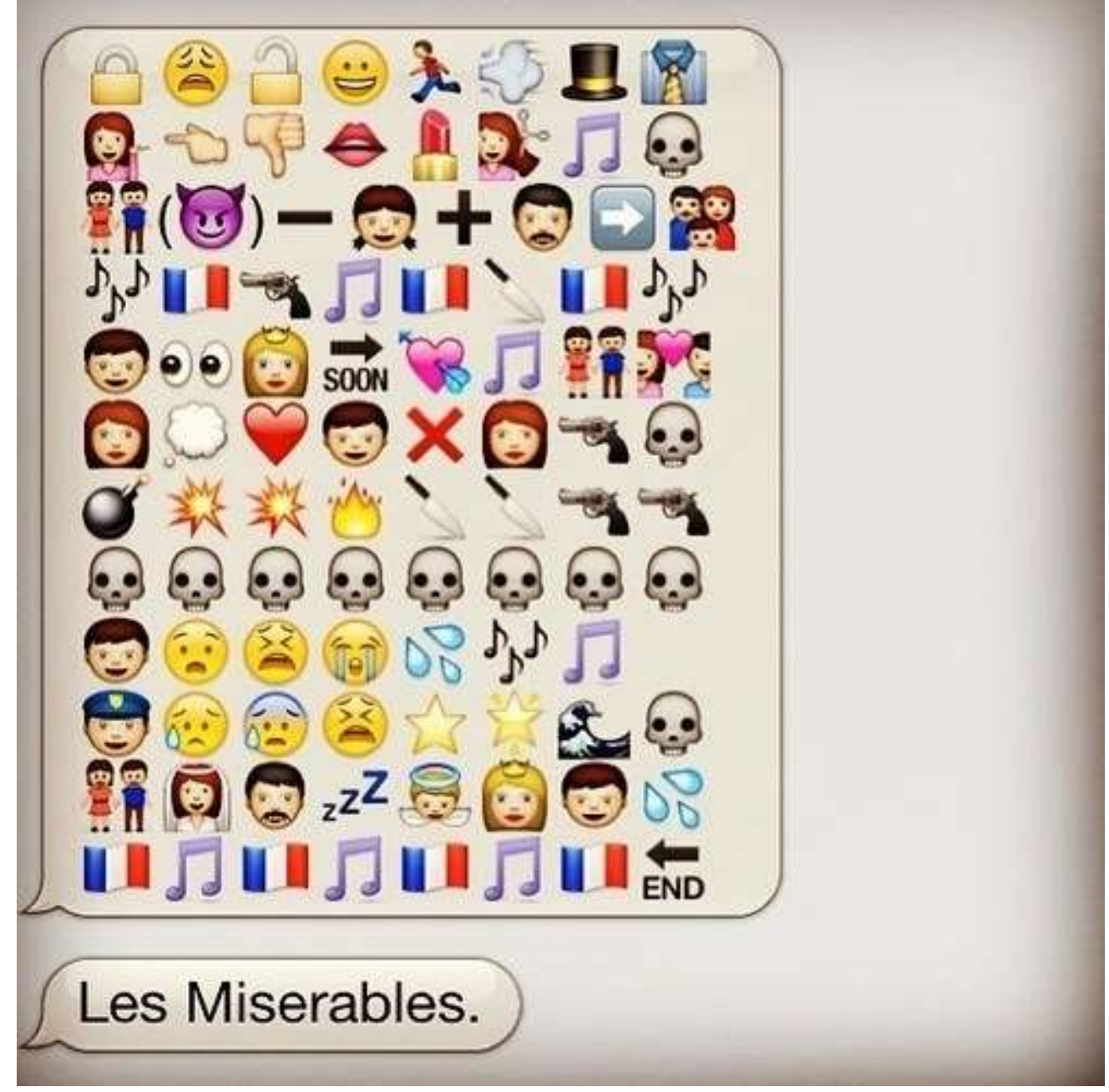

Figure 14. Les Miserables in Emoji

Source: https://www.buzzfeed.com/kdries/23-famous-movies-and-songs-reenacted-in-emojis-4fsn, (2016)

A similar approach is taken when describing the plot of "The Notebook" as the figure below illustrates.

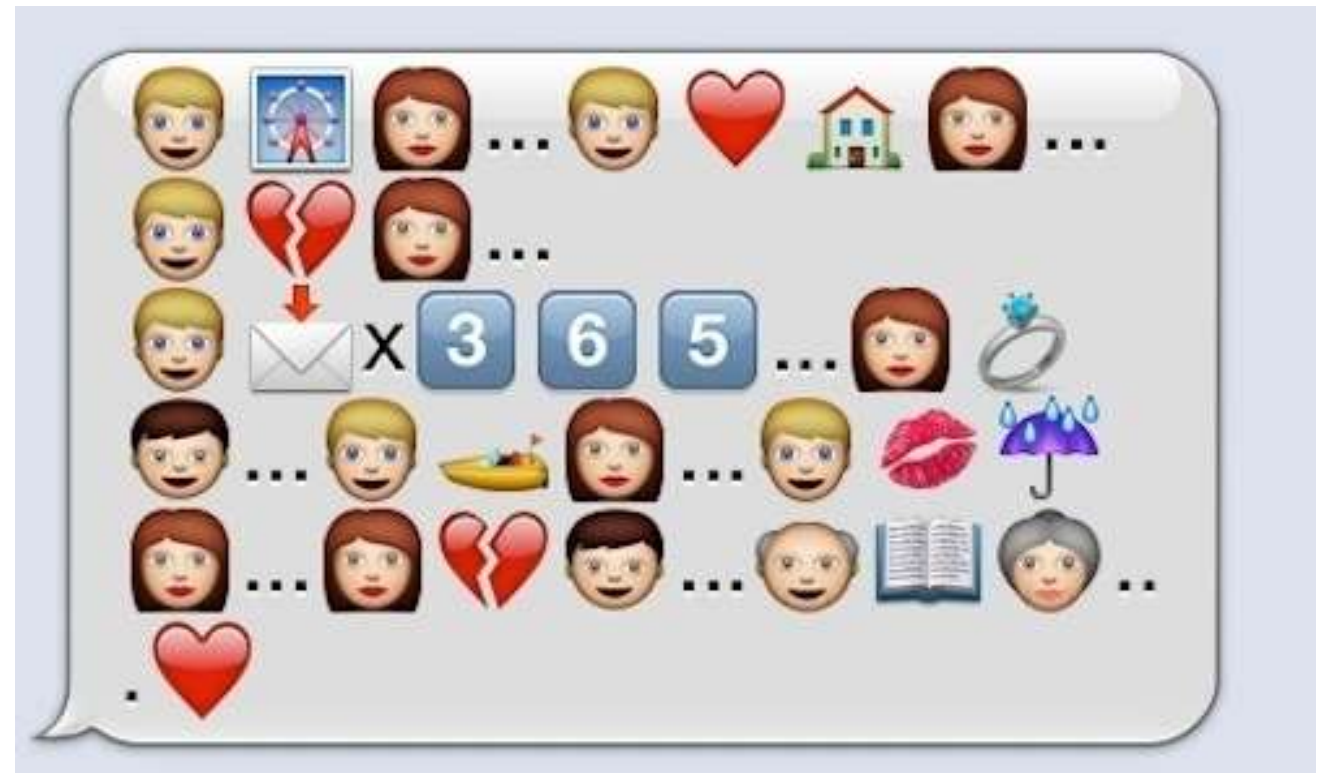

Figure 15. Plot of The Notebook in Emoji

Source: https://www.buzzfeed.com/kdries/23-famous-movies-and-songs-reenacted-in-emojis-4fsn, (2016) 
There is a clear parallel here to ancient pictographic representations of battles and other historical stories demonstrating again that emojis are not a new language, but an evolution of older communication systems.

In this respect, they have also been adopted by business, as the tweet in the figure below from the bank Goldman Sachs illustrates.

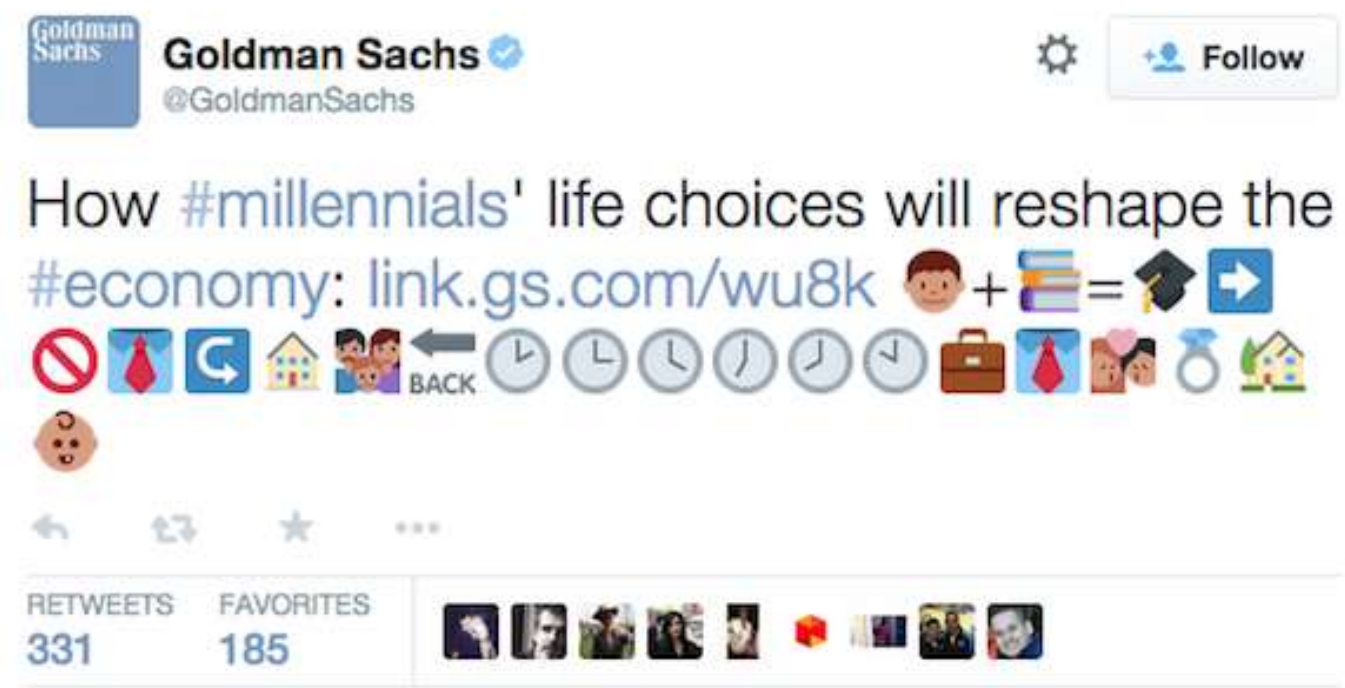

11:56 AM - 6 Mar 2015

Figure 16. Emoji Laden Tweet from Goldman Sachs

Source: https://www.buzzfeed.com/kdries/23-famous-movies-and-songs-reenacted-in-emojis-4fsn, (2016)

What this tweet further underlines is that emojis are not just for young teenagers or millennials, they are used widely in business and advertising as firms recognise the value of visual representations and connections in digital language. Indeed, Twitter now produces a "real time" emoji tracker so that users can see which emojis are being the most utilised at any given time as the figure below indicates.

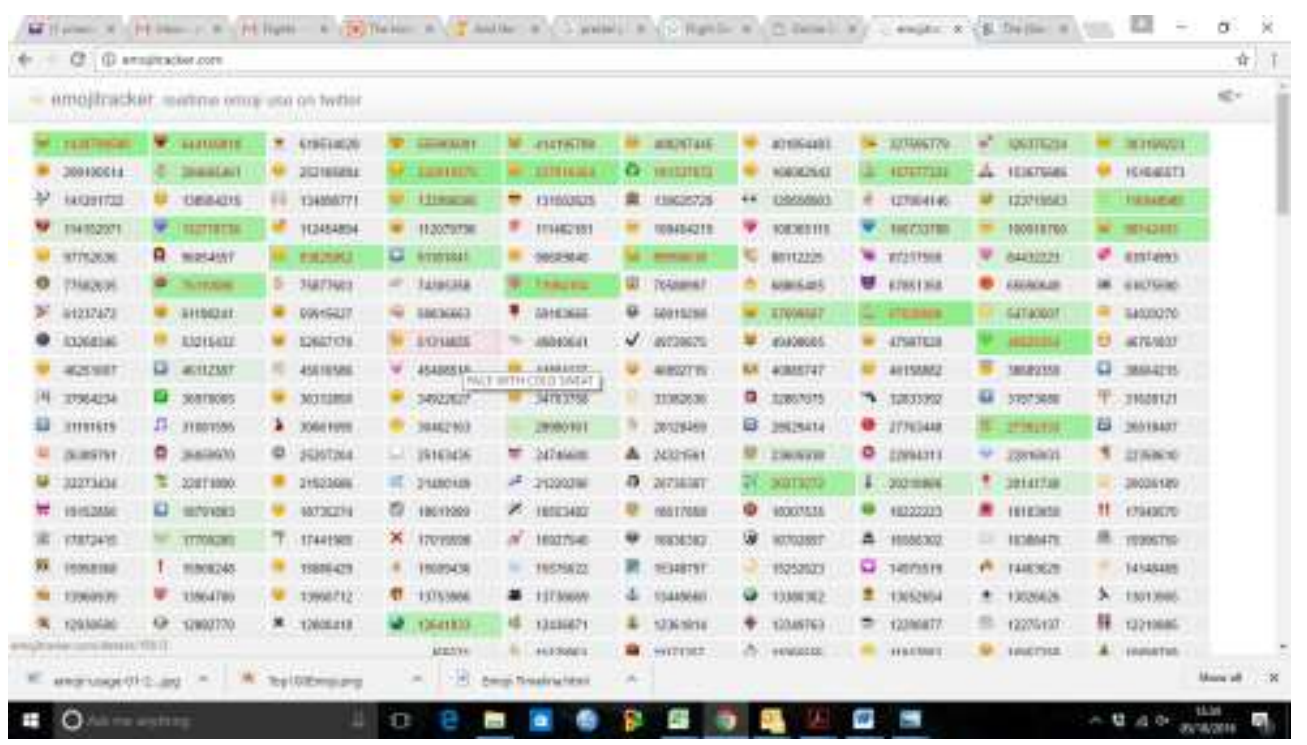

Figure 17. Twitter Real Time Emoji Tracker

Source: http://emojitracker.com/, (2016)

These examples of emoji use clearly demonstrate not only the popularity and ubiquitous nature of these images but also that they are developing as a real form of language. At the same time, these images also demonstrate the correlation to older forms of pictorial language and represent a recognition of the positive way that people view visual communication, particularly in the digital domain. 